\title{
RESEARCH CHALLENGES IN TEXT MINING AND EMPIRICAL RESEARCH DIRECTIONS
}

\author{
K Ranjith Reddy \\ Research Scholar, Department of CSE, Madhav University, Abu Road, \\ Sirohi, Rajasthan 307026, India \\ ranjith.kssr5@gmail.com \\ Dr. Sanjay Chaudhary \\ Professor, Department of CSE, Madhav University, Abu Road, \\ Sirohi, Rajasthan 307026, India \\ schaudhary0020@gmail.com
}

\begin{abstract}
Document categorization is one among the prime successive and fundamental issues in viewpoints of information examination, with applications from data recovery as well as spam sifting to content personalization and etymological communication content measure. Automated text order classification is an especially difficult assignment in present day information investigation, both from an observational and from a hypothetical viewpoint. This issue is of focal interest in numerous web applications, and therefore it has received consideration from specialists in such assorted zones. Quickly streaming surges of text are created by online news, web-based media and perpetual various applications, along with subsequently the need to precisely and adaptively sort them into the sub-streams could be a significant one. The emphasis on exclusively making utilization of delimited resources could be a result of size of particular streams: each time and memory should be held under the influence. The economical analysis of the huge datasets is the one among the most challenges in trendy machine intelligence and data processing applications. In this paper, we extensively surveyed significant developments occurred in this domain over past years. We have listed some significant existing methods, tools, standard datasets for performing text mining and analysis. We also given an argument on the various open challenges involved in this domain along with the problem identification and our possible research directions / objectives to overcome these challenges.
\end{abstract}

Keywords: Text classification; Information retrieval; Machine learning; Feature selection; Language models.

\section{Introduction}

Learning sensible formulations of text plays a crucial role in several linguistic communication process / Natural Language Processing tasks, like docs grouping, positioning, sentimental investigation, etc.80-90\% of all content information is held in different unstructured configurations. Helpful data can be obtained from this unstructured, raw information. Extraction of interesting information (or patterns) from this kind of data is - Text Mining.[1][2] Intelligence in text mining is based on NLP techniques. Since 10 years, abundant measure of text information is being produced through different web sources in on the web or on the other hand disconnected situations. This immense measure of information is basically conflicting and non-organized organization, so difficult to measure through processing machines accessible. With the appearance of super computers and the evolving data age, measurable and logical issues have additionally developed both in the size and intricacy. Procedures for the arrangement of text archives can be splitted into the two expansive classes of supervised alongside unsupervised learning systems. Supervised technique takes an item, it is ordinarily a vector as an information and yields an ideal value.[3] In unsupervised categorization technique, countless obscure items can be inspected. Text categorization can likewise be thought as the instrument to offer labels to different regular corpus text archives. 


\subsection{General Text Mining Framework.}

The general text mining framework is given as Fig 1. In that, first the data cleaning and preprocessing steps are performed for the corpus which consists of steps like - tokenization, whitespace removal, normalization, stopwords removal (e.g., "a", "an"," the"," in" etc.), as these are considered as useless while text modeling, punctuation symbols removal, stemming, lemmatization etc. Next, the dataset is partitioned into training and validation dataset segments. Then the features extraction process is performed where for each training instance, labels are given. In text documents, each word can act as feature. Then a learning algorithm is applied in these feature vectors which will result to a model (also called as text classifier). The performance is judged on validation data and misclassification rate is computed. Finally, the optimal model is deployed and the predictive analysis is performed on test data.

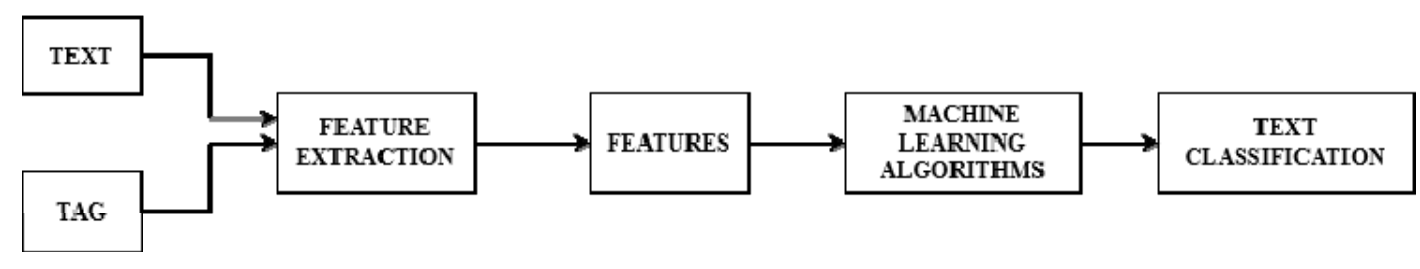

Fig 1. General Framework Flow

\subsection{Text Mining Applications}

Text arrangement includes a learning strategy whose applications are in the empirical spaces like -

(1) Information retrieval: Data Retrieval is perceived as a completely programmed measure that reacts to a client inquiry by analyzing an assortment of archives and returning an arranged record list that ought to be pertinent to the client prerequisites as communicated in the question. The movement of getting data assets pertinent to a data need from an assortment of data assets. Searches can be founded on metadata or on fulltext ordering.

(2) Language identification: Language distinguishing is the issue of deciding the regular language that a document or part thereof is written in. Programmed language ID has been broadly investigated for more than fifty years. Today, language ID is a critical piece of numerous content preparing pipelines, as text handling procedures by and large expect that the language of the info text is known.

(3) Opinion mining and Sentiment Analysis: Assessment mining is the study of utilizing text examination to comprehend the drivers behind open supposition. All content is naturally minable. Accordingly, while web-based media might be a conspicuous wellspring of current assessment, audits, call focus records, website pages, online discussions and study reactions would all be able to demonstrate similarly useful. Sentiment analysis - an archetype to the field of assessment mining - looks at how individuals feel about a given subject (be it good or contrary), assessment mining goes a level further, to comprehend the drivers behind why individuals feel the manner in which they do.

(4) Spam filtering: Spam channels distinguish spontaneous, undesirable, and infection pervaded email (called spam) and prevent it from getting into email inboxes. Network access Providers (ISPs) use spam channels to ensure they aren't circulating spam. Spam channels use "heuristics" strategies, which implies that each email message is exposed to a large number of predefined rules (calculations). Each standard appoints a mathematical score to the likelihood of the message being spam, and if the score passes a specific limit the email is hailed as spam and hindered from going further. This scenario is represented as Fig 2 


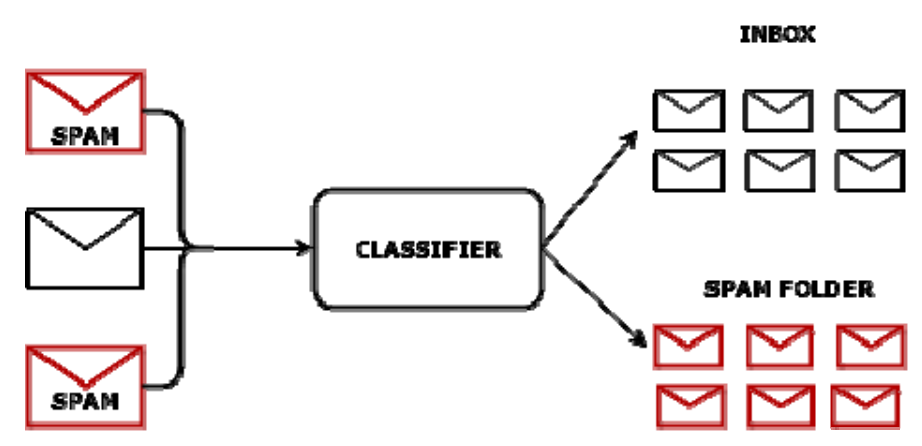

Fig 2. Automated Spam Filtering

(5) News article classification: Text archives are perhaps the most extravagant wellspring of information for organizations: regardless of whether looking like client assistance tickets, messages, specialized records, client audits or news stories, they all contain significant data that can be utilized to robotize moderate manual cycles, better get clients, or discover important bits of knowledge. Notwithstanding, customary calculations battle at preparing these unstructured records, and this is the place where AI assumes a crucial part.

(6) Webpage classification: Categorization of Web page content is vital for some errands in Web data recovery, for example, keeping up Web registries and centered slithering. The uncontrolled idea of Web content presents extra difficulties to Web page arrangement when contrasted with customary content characterization, yet the interconnected idea of hypertext additionally gives includes that can help the process modules.

(7) Automated QA's: Even with modern search engines, there are many scenarios where the users struggle to find out the information they are investigating for. This is especially true when the information need is complex, and when the user is unable to distill down their information need into a few keywords. This is where, the Automated question answering procedure is developed. Chatbots is an example of this scenario.

(8) Text Summarization: Programmed text synopsis is a typical issue in AI and natural language preparing (NLP). Text summarization alludes to the strategy of shortening long bits of text. The goal is to make an intelligent and familiar rundown having just the primary concerns laid out in the record.

(9) Creating suggestion and recommendations: Using past customer purchases, page views, reviews, and demographic info, the sites (like Amazon, Flipkart etc.) offer targeted recommendations for cross-sells and up-sells.

\subsection{Organization of the paper}

Remaining portion of the paper is organized as: Section2 provides an extensive review of methods developed over past years. Section 3 presents some significant definitions and statistical preliminaries. Existing text mining methods along with their advantages and drawbacks are summarized in section 4. Some significantly used tools and standard text data corpuses are given in section 5. Challenges in this domain and research objectives are given in section 6 . Finally, section 7 presents conclusive summary. 


\section{Literature Review}

This section reviews some significant literature in this domain.

A.L. Blum et al. [4] suggested some features engineering methods. Authors in [5 - 10] given various empirical study, methods for feature selection and metrics for text classification. Y. Yang et al. [11 - 12] given Sampling strategies, comparative study and learning efficiency in text categorization process. H. Kim et al. [13] presented dimension reduction into the text labelling with the help of support vector machines. E.Leopold et al. [14] describes representation of texts in input space while applying SVM. F. Sebastiani [15] given some efficient Machine learning approaches in automated text categorization.

Y. Yang et al. [16 - 17] proposed Noise reduction based statistical approach towards text categorization. Zhang et al. [18 - 19] analyzed the power of regularized linear natured categorization techniques in text arrangement. Yuchen et al. [20] given Macro Grammars along with Holistic Triggering for an efficient semantic scanning of text records. Yiming Li et al. [21] in their methodology embraced neural organization for text portrayal and handling. Schuster et al. [22] given Manning Gapping archives.

To subsequently improve the order and further expectation precision for any broad content classifier is a critical issue and much measure of exploration has been done around here. In 1998, Lam and Ho [23] has proposed an instrument to set the archive proptotype vectors for different categories. In 2007, Tang and Gao [24] suggested a methodology to improve the characterization precision. In this, he joined the k-closest neighbor and support vector machine draws near. Sarkar M.(2007) [25] proposed the computationally fluffy way to deal with improve the characterization precision of KNN strategy. This technique requires an extensively enormous space to store the preparation and testing datasets.

Liu et. al.(2002) [26] given a methodology to tackle text categorization issue. In 2004, Yu et. al. [27] introduced a strategy, in which for building a classifier, support vector machine (SVM) prototype is used. Y. Li et. al. (2006) [28] proposed a component utilizing RS-oriented reasoner. It improves the TC precision, diminishes the quality term space intricacy. Duoqian Miao et. al.(2009) [29] given a content categorization half breed approach dependent on RST. Weibin Deng (2011) [30] proposed a sort of cross breed methodology for TC dependent on RST. Essentially two phases are available in this calculation. In first stage, practically all archives are categorized into a few classes, Naive Bayes technique is used for grouping. Later they given the comparative execution examination for both various stages. Sadiq et. al. (2012) [31] recommended a framework for record portrayal alongside their arrangement. After the prepreparing stemming measure and so forth, text categorization model is assembled. They have utilized the directed order. They have investigated the exhibition of the framework. In 2013, Guansong Pang et. al. [32] proposed the TC procedure dependent on Generalized Cluster Centroid(GCC) similarity. Here, they have incorporated two notable classifier. They used a grouping calculation to accelerate the KNN classifier. Basant Agarwal and Namita Mittal (2013) [33] have introduced a cross breed credits choice technique dependent on InformationGain (IG) for particularily notion arrangement. They played out the examinations on standard datasets. Ricardo et. al. (2014) [34] given an effective philosophy for multi-named corpus. Comparison of proposed model with other classifiers is likewise introduced. The examination of a few Semantic Text Based Categorization systems is yielded (2014) Nibaran Das et. al. [35]. Zhu el. al. (2015) [36] proposed group investigation technique for CBR. Investigations are performed on UCI datasets. Jun Wang et. al. (2015) [37] recommended a high level methodology for pages order. V K Bhalla et. al. [38] (2016) uses "SVM model". The proposed conspire outflanks well in tests. Abdullah Saeed Ghareb et. al. (2016) [39] suggests a nature wise half and half FS technique for TC, that depends on upgraded GA. This methodology deals high dimensionality of reports during highlight choice and later plays out the categorization stage. 


\section{Definitions and Statistical Preliminaries}

\subsection{Definitions and statistical preliminaries}

(1) Tokenization: It is an early advance in the NLP interaction, a stage what parts longer strings of text into more modest pieces, or tokens. Further handling is for the most part performed after a piece of text has been suitably tokenized. The scenario is represented as Fig 3.

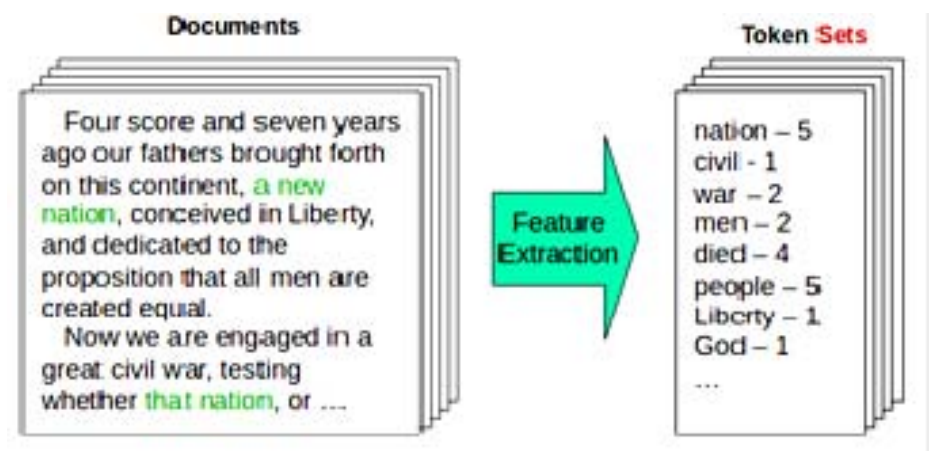

Fig 3. Tokenization Scenario

(2) Normalization: Standardization is arrangement of related assignments intended to put all content on a level battleground: changing all content over to a similar case (upper or lower), eliminating accentuation, extending withdrawals, changing numbers over to their promise counterparts, etc. Standardization puts all words on equivalent balance, and permits preparing to continue consistently.

(3) Stemming: Stemming is the way toward eliminating appends (suffixed, prefixes, infixes, circumfixes) from a word to get a word stem. For example - [Playing, Played] $\rightarrow$ Play

(4) Lemmatization: lemmatization is identified with stemming, contrasting in that particular lemmatization can statistically absorb authoritative oriented structures dependent on a word's lemma.

For example - [Better, Best ] $\rightarrow$ Good

(5) Stop Words: Stop words are those words which are sifted through before additional preparing of text, since these words contribute little to by and large significance, given that they are by and large the most widely recognized words in a language. For instance," the"," and"," a", etc.

(6) Corpus: Corpus depicts to an assortment of texts. Such assortments might be framed of a solitary language of writings, or can traverse different dialects. Corpora comprise of themed messages (authentic, Biblical, and so on) Corpora are by and large exclusively exploited for factual semantic examination and theory testing.

(7) Bag of Words: Bag of words is a specific portrayal model used to work on the substance of a determination of text. The sack of words model excludes language structure and word request, however is keen on the quantity of events of words inside the content.

Example text: "good, good", said Teacher.

The resulting BOW (bag of words) representation as the dictionary \{'good': 2, 'said': 1, 'Teacher': 1 \}

(8) n-grams: n-grams is another portrayal model for improving on text choice substance. Instead of the orderless portrayal of pack of words, n-grams demonstrating is keen on saving adjoining successions of $\mathrm{N}$ things from the content choice. 
An illustration of trigram (3-gram) model for the above model (" good, good", said Teacher) shows up as a rundown portrayal underneath

$$
\begin{aligned}
& \text { \{"good good said", } \\
& \text { "good said Teacher" }\}
\end{aligned}
$$

(9) Parts-of-speech (POS) Tagging: POS labeling comprises of allotting a classification tag to the tokenized parts of a sentence. The most mainstream POS labeling would distinguish words as things, action words, adjectives, and so on.

(10) Statistical Language Modeling: It is the way toward building a statistical language model which is intended to give a gauge of a characteristic language. For an arrangement of information words, the model would allot a likelihood to the whole grouping, which adds to the assessed probability of different potential successions.

(11) Regular Expressions: Regular Expressions( regexp or regex), are a time-tested strategy for compactly portraying examples of text. A normal articulation is addressed as an uncommon content string itself, and is intended for creating search designs on choices of text.

(12) Significant Statistical Performance Measures: Assume,we are thinking about two classes(categories), additionally called binary classifier, at that point the confusion matrix looks like below.

\begin{tabular}{|c|l|l|}
\hline \multirow{2}{*}{$\begin{array}{c}\text { Actual } \\
\text { Class }\end{array}$} & \multicolumn{2}{|c|}{ Predicted Class } \\
\cline { 2 - 3 } $\mathrm{P}$ & \multicolumn{1}{|c|}{$\mathrm{P}$} & \multicolumn{1}{c|}{$\mathrm{N}$} \\
\hline & True & False \\
& Positives & Negatives \\
& $(\mathrm{TP})$ & $(\mathrm{FN})$ \\
\hline $\mathrm{N}$ & False & True \\
& Positives & Negatives \\
& $(\mathrm{FP})$ & $(\mathrm{TN})$ \\
\hline
\end{tabular}

Table 1. Confusion Matrix

Here, the two specific classes are being denoted as $-\mathrm{P}$ and N. Let's suppose, there are 51 instances for documents classification. Out of those, 22 instances are falling in (TP), 9 instances are falling in (FN), 7 instances are falling in (FP), 13 instances are falling in $(\mathrm{TN})$. So of course, $(22+9+7+13)=51$ total instances. Now,

\section{Cohen's Kappa measure:}

From above $\mathrm{TP}=22, \mathrm{FN}=9, \mathrm{FP}=7, \mathrm{TN}=13$,

Total Instances $(\mathrm{TI}) \quad \mathrm{TI}=(\mathrm{TP}+\mathrm{FN}+\mathrm{FP}+\mathrm{TN})=22+9+7+13=51$

Ground truth: GP $(22+7=29)$, GN $(9+13=22)$

ML classifier: MP $(22+9=31), \mathrm{MN}(7+13=20)$

Calculate, Observed accuracy (OA): $O A=\frac{T P+T N}{T I}$

By (1) Observed accuracy is $\quad O A=\frac{22+13}{51}=\frac{35}{51}=0.69$ 
Expected Accuracy (EA):

By (2) Expected accuracy is
$E A=\frac{\left(\frac{(G P \times M P)}{T I}+\frac{(G N \times M N)}{T I}\right)}{T I}$

$$
E A=\frac{\left(\frac{(29 \times 31)}{51}+\frac{(22 \times 20)}{51}\right)}{51}=0.51
$$

Kappa measure can be computed with formula $\frac{O A-E A}{1-E A}$

Precision -It is the ratio of correctly predicted positive observations to the total predicted positive observations.

$$
\text { Precision }=\frac{T P}{T P+F P}
$$

Recall (sensitivity)- It is the ratio of correctly predicted positive observations to the all observations in actual class $P$.

$$
\text { Recall }=\frac{T P}{T P+F N}
$$

F1 score - It is the weighted average of above two measures.

$$
\text { F1 score }=\frac{2 \times(\text { Re call } \times \text { Pr ecision })}{\operatorname{Re} \text { call }+ \text { Pr ecision }}
$$

(13) Machine Learning: Machine Intelligence is the point at which a PC has been instructed to perceive designs by giving it information (or preparing information) and a calculation which helps in understanding that information. We allude this interaction of learning as 'preparing' and the yield of this cycle is alluded as a 'model'. The model is taken care of with new information (or test information) and it can reason about this new data dependent on what it has recently realized.

AI models decide a bunch of rules utilizing tremendous measures of registering power that a human cerebrum would be unequipped for preparing. The more information an AI model is taken care of, the more intricate the principles - and the more precise the forecasts. To sum up, the objective of AI is to comprehend the design of information so exact forecasts can be made dependent on the properties of that information. While a measurable model is probably going to have an innate rationale that can be perceived by the vast majority, the standards made by AI are frequently outside human ability to understand in light of the fact that our minds are unequipped for processing and investigating huge informational indexes.

(1) Supervised learning: Deals cause the capacity to gain from the accessible preparing part of dataset. A managed learning calculation misuses the accessible preparing information part and makes a derived capacity, which can be then abused further for planning new ones. Different directed learning calculations are accessible, for example, Support Vector Machines, Neural Networks and Naïve Bayes classifiers and so on.

(2) Unsupervised learning: Manages unlabeled information without taking any already characterized dataset for model preparing. Unsupervised learning can be thought as an intense apparatus for search for examples and drifts and dissecting accessible information. There are different methodologies utilized by 
unsupervised learning for example K-implies clustering, progressive grouping, self-organizing maps and so forth. Other numerous learning types are -

- Active learning

- Kernel-based learning

- Transfer learning

- Distributed learning

- Association rule learning

- Inductive logic programming

- Reinforcement learning

- Similarity and metric learning

(14) Feature Engineering: Feature Engineering is an interaction of changing crude information into include vector which helps in expanding the prescient force of AI calculations. It is the main craftsmanship in AI which makes the enormous contrast between a decent model and an awful model. For instance Suppose we are given the scope, longitude and other information with the given name "Cost of House". We need to foresee the cost of the house around there. The scope and longitude are not of any utilization on the off chance that they are separated from everyone else. Thus, here we will utilize the crossed section include designing. We will join the scope and the longitude to make one element. Joining into one component will assist the model with learning.

(15) Singular Value Decomposition (SVD): Vectors addressing records and queries are projected in new, low dimensional space acquired by shortened SVD. By applying the SVD on a term-archive network, reports will be changed in a vector space of counterfeit ideas. Every one of the k diminished measurements compares to a dormant idea which serves to separate the documents.

(16) Dimensionality and Heterogeneity of data: Dimensionality in AI alludes to the number of features are available in dataset. At the point when the dimensionality builds, the volume of the space increments so quick that the accessible information become scanty. The scourge of dimensionality discloses to us that assessing a few amounts gets more enthusiastically as the quantity of measurements of an informational index increments - as the information gets larger or more extensive. For instance, medical care information is infamous for having huge measures of factors (for example pulse, weight, cholesterol level). In an ideal world, this information could be addressed in an accounting page, with one section addressing each measurement. Practically speaking, this is hard to do, partially on the grounds that numerous factors are between related (like weight and circulatory strain). A heterogeneous populace or test is one where each part has an alternate incentive for the trademark you're keen on. For instance, patients are commonly an exceptionally heterogeneous populace as they vary with numerous variables including socioeconomics, demonstrative test outcomes and clinical accounts and so forth.

(17) Bias and Variance: Bias implies how distant our expectations are from genuine qualities. The blunder because of predisposition is taken as the contrast between the normal (or normal) expectation of our model and the right worth which we are attempting to anticipate. Obviously, we just have one model so discussing expected or normal expectation esteems may appear to be somewhat bizarre. Notwithstanding, assume we could rehash the entire model structure measure more than once: each time we assemble new information and run another investigation making another model. Because of irregularity in the hidden informational indexes, the subsequent models will have a scope of forecasts. In this manner, inclination marks how far away overall these models' forecasts are from the right value. The mistake because of fluctuation is taken as the inconstancy of a model expectation for a given information point. Once more, envision we can rehash the whole model structure measure on various occasions. The change is how much the forecasts for a given point fluctuate between various acknowledge of the model.

(18) Confusion Matrix: A confusion matrix is a N X N utility, where $\mathrm{N}$ is the amount of classes being expected, used to evaluate the display of a request model (or"classifier") on a lot of test data for which the authentic characteristics are known. It has four distinct blends of anticipated and genuine qualities as demonstrated in Table 2. 


\begin{tabular}{|c|c|c|}
\hline \multirow{2}{*}{$\begin{array}{c}\text { Predicted } \\
\text { Values }\end{array}$} & \multicolumn{2}{|c|}{ Actual Values } \\
\cline { 2 - 3 } & Positive (1) & Negative (0) \\
\hline Positive (1) & TP & FP \\
\hline Negative (0) & FN & TN \\
\hline
\end{tabular}

Table 2. Confusion matrix parameters

Here,

True Positive (TP): means we predicted positive and it's true.

False Positive (FP): means we predicted positive and it's false.

True Negative (TN): means we predicted negative and it's true.

False Negative (FN): means we predicted negative and it's false.

Confusion Matrix is extremely helpful in measuring Recall, Precision, Specificity and Accuracy

(19) Dimensionality Reduction: It is generally achieved through two types of methodologies

i.e. - (i) dimension reduction based on multidimensional projection planes for data.

(ii) dimension reduction based on optimal features selection.

Dimension reduction is advantageous in terms of mainly speed-up as it diminish unique information, for example to discover insignificant arrangements of information with a similar information as in the original data

\section{Existing Text Analysis and Classification Methods}

\subsection{Existing Text Analysis and classification methods}

Various statistical machine learning based text analysis and classification methods are evolved over past years which are summarized here.

- Bayesian Classifiers: In these kinds of classifiers, the measurable data and probabilistic information is utilized for metadata creation. Here, Bayes' hypothesis is utilized with basic autonomy guesses among features. Since 1950's, it is ceaselessly examined. It is having its applications in - clinical conclusion examination, spatial imaging information, text arrangement and so forth. This classifier is profoundly versatile and it requires various boundaries which are straight in number of variable indicators in parts of learning problem.

- Artificial Neural Network: It is an assortment of classifier, whose standard plan design and usefulness is fairly like human mind structure algorithmic model. For arrangement question, the specific piece of neural organization changes. To start with, in the preparation, the topological design and number of organization hubs present in the center layer are resolved. In contrast to SVM, it has no quirk for example n-dimensional planes and hyperplanes. In any case, preparing of informational indexes measure here is time taking, delivers less exact and proficient outcomes too.

- Fuzzy Support vector machine: In FSVM, each individual preparing point has a place precisely with close to one specific class. Any focuses which forces commotion, couldn't be ordered by SVM. In this way, they are managed here through FSVM. Pre-information data about datasets is required, as stochastic and probabilisticdata. Here, a few stochastic relationships can be recognized.

- Decision Trees: A Decision tree is a design that fuses a root hub, branches, and leaf hubs. Each inside hub signifies as a test on a property, each branch suggests the result of a test, and each leaf hub contains a class label. Root hub is the highest hub in the tree. In 1980, J. Ross Quinlan proposed a decision tree calculation named as ID3(Iterative Dichotomiser). Further, He proposed an augmentation of ID3. This calculation follows the covetous methodology. No backtracking is accessible in this computation. 
- Random Forest: Random forest resembles bootstrapping calculation with Decision tree (CART) model. Say, we have 1000 perception in the total populace with 10 factors. Arbitrary backwoods attempts to construct numerous CART models with various examples and diverse introductory factors. For example, it will take an arbitrary example of 110 perception and 10 haphazardly picked beginning factors to fabricate a CART model.

- Convolutional Neural Network: In Convolutional neural networks, convolutions over the information layer are utilized to figure the yield. This outcomes in nearby associations, where every area of the information is associated with a neuron in the yield. Each layer applies various channels and joins their outcomes.

- Recurrent Neural Network: Dissimilar to Feed-forward neural networks in which actuation yields are engendered uniquely one way, the initiation yields from neurons proliferate in the two ways (from contributions to yields and from yields to contributions to) Recurrent Neural Networks. This makes loops in the neural networks engineering which goes about as a 'memory condition' of the neurons. This state permits the neurons a memorable capacity what have been realized up until now. The memory state in RNNs gives a benefit over customary neural networks yet an issue called Vanishing Gradient is related with them. In this issue, while learning with countless layers, it turns out to be truly difficult for the organization to learn and tune the boundaries of the prior layers.

- Rule-based Approaches: Rule-based methodologies order text into coordinated gatherings by utilizing a bunch of high-quality phonetic guidelines. These guidelines educate the framework to utilize semantically important components of a book to distinguish pertinent classifications dependent on its substance. Each standard comprises of a forerunner or design and an anticipated classification.

\subsection{Advantages and Limitations}

The advantages and some limitations of most significantly used text classifiers are summarized in table 3 .

\begin{tabular}{|c|c|c|}
\hline Method & Advantages & Limitations \\
\hline $\begin{array}{l}\text { Shallow } \\
\text { Neural Nets }\end{array}$ & $\begin{array}{l}\text { - It is self-adaptive procedure. } \\
\text { - Having a distributed memory. } \\
\text { - Parallel processing capability. }\end{array}$ & $\begin{array}{l}\text { - Data sets training process is time } \\
\text { consuming. } \\
\text { - It's a complex procedure to choose } \\
\text { network topology. } \\
\text { - Hardware dependence }\end{array}$ \\
\hline $\begin{array}{l}\text { Support } \\
\text { Vector } \\
\text { Regression }\end{array}$ & $\begin{array}{l}\text { The decrease in the computational } \\
\text { complexity for SVM classifier is } \\
\text { possible. } \\
\text { - In the selection of threshold, flexibility } \\
\text { is present. } \\
\text { - Error factor calculation is easy. }\end{array}$ & $\begin{array}{l}\text { - Complexity is more to completely } \\
\text { understand the structure. } \\
\text { - Choosing optimal kernel is hard. }\end{array}$ \\
\hline $\begin{array}{l}\text { Bagged } \\
\text { Modelling }\end{array}$ & $\begin{array}{l}\text { - Reduces variance. } \\
\text { - Mostly avoids overfitting. } \\
\text { - Improves the stability and accuracy. }\end{array}$ & $\begin{array}{l}\text { - It can sometimes degrade the } \\
\text { performance of stable methods } \\
\text { such as K-nearest neighbors. }\end{array}$ \\
\hline Fuzzy SVM & $\begin{array}{l}\text { - Fuzzy methods used on the SVM solve } \\
\text { the problem that the SVM is sensitive to } \\
\text { the outliers or noises in the training set. }\end{array}$ & $\begin{array}{l}\text { - Pre-information about data sets } \\
\text { e.g. - stochastic and probabilistic } \\
\text { information is required. }\end{array}$ \\
\hline $\begin{array}{l}\text { Decision } \\
\text { Tress }\end{array}$ & $\begin{array}{l}\text { - One of the most useful aspects of } \\
\text { decision trees is that they force you to } \\
\text { consider as many possible outcomes of a } \\
\text { decision as we can think of. }\end{array}$ & $\begin{array}{l}\text { - Small change in the data can lead } \\
\text { to a large change in the structure of } \\
\text { the optimal decision tree (unstable } \\
\text { behavior). }\end{array}$ \\
\hline
\end{tabular}




\section{Tools and Standard Text Data Sets}

\subsection{Text Mining and Analysis Tools}

Although constructing an information retrieval (IR) and knowledge discovery (KD) system is a difficult task, there has been significant recent progress in using machine learning methods, tools to help automate the construction of IR and KD systems Some significantly used tools and standard text datasets are discussed in this section. Tools help users to gain proper insights from text data in order to plan and act accordingly. Some of the significantly used tools for text analysis are listed below.

- RapidMiner - It is a software platform that provides an integrated environment for data preparation, machine learning, text mining and predictive analytics.

- GATE- General Architecture for Text Engineering (GATE) is a suite of tools, used for human language processing and information extraction.

- KHCoder - It is an open source software for particularly quantitative content analysis and text mining.

- RStudio- It is a free and open-source integrated development environment for R, a programming language for statistical computing and data analysis.

- Visual Text - It is the premier IDE for building information extraction systems, natural language processing systems.

- Natural Language Toolkit- NLTK is a suite of libraries and programs for symbolic and statistical natural language processing for English written in the Python language.

- Datum box -It offers a large number of off-the shelf Classifiers and Natural Language Processing services.

- Carrot2-It can automatically cluster small collections of documents, e.g search results or document into thematic categories.

- LingPipe-It is toolkit for processing text using computational linguistics.

- Gensim-It is an open-source library for unsupervised topic modeling and natural language processing, using modern statistical machine learning.

- tm-package-It is a framework for text mining applications within R.

- Aika-Aika (Artificial Intelligence for Knowledge Acquisition) is an artificial neural network designed specifically for the processing of natural language texts. A key feature of the Aika algorithm is the ability to evaluate and process various interpretations of the individual sections of a text. Aika combines several ideas and approaches from the field of AI such as artificial neural networks, frequent pattern mining and logic-based expert systems.

\subsection{Standard Text Data Sets}

Some of the open-source standard text datasets and repositories are listed here which can be used for experimentation and model simulation purpose.

(1) IMDB Movie Reviews dataset

(2) HotspotQA dataset

(3) Amazon Reviews dataset

(4) E-mail Spam dataset

(5) 20 Newsgroup dataset

(6) BBC dataset

(7) UCI ML Repository

(8) Reuters- 21578 corpus

\section{Research Directions}

This section discusses about the problems and challenges in this domain along with the possible research directions / objectives.

\subsection{Problems and Challenges}

The open problems and challenges involved in this research domain are listed as follows -

- Presence of unnecessary and highly correlated variables in the text corpus. 
- Insufficient collected data (sparse natured) from documents.

- Curse of dimensionality of text data - suffers from memory fitting problem while analyzing (computationally expensive).

- Data may be imbalanced of may posses dynamic nature.

- Data obtained from heterogenous sources.

- Diversity in statistical distribution while predicting is a key challenge.

- Presence of outliers in the collected data.

- Incompleteness (missing values presence) in observations.

- Inconsistencies (uncertainty amount) present in the data.

- Dependence on discrete natured data is also a challenge for text classification and computational language modeling.

\subsection{Research Objectives}

The research objectives towards overcoming the existing key challenges are as follows -

(1) Text classification encounters the major difficulty of the high dimensionality of text features / variable vectors and available unstructured text data. Therefore, a dimension reduction technique is very much required to discard irrelevant features from the feature set vector. In our research work, we develop new soft computing and applied statistics-based approach to address this issue and test their performance using standard text datasets /corpuses from repositories, discussed in section 5.2.

(2) We adopt and develop conceptualization for dimension reduction method based on multidimensional projection planes for data and dimension reduction based on optimal features selection from text corpus.

(3) Further, we develop a text classification framework where the optimally minimal set of chosen feature vectors / variables will be utilized to perform language modeling on the particular text data (corpus).

(4) To prove the novelty of our proposed frameworks, we perform validation and testing of developed model also perform comparative analysis with the significant existing approaches.

(5) The developed text analysis frameworks will reflect the computational benefits as -

- Developed methodologies will be able to model complex functions, dealing with uncertainty, provide enhanced learning and generalization capabilities.

- It allows to diminish unique content information, for example to discover negligible arrangements of information with the equivalent information as in the first corpus which gives the advantages of better speed up.

- It needn't bother with any preliminary or extra data about information, as - likelihood in insights, entropy measure and grade of participation.

- It will be efficient for finding hidden patterns in text data and extract knowledge from them.

- Due to granular and non-dependance nature of computations involved, it is suited for concurrent (parallel/distributed) processing.

- It offers straightforward interpretation and better visualization of obtained results.

\section{Conclusion}

Learning sensible representations of text plays a crucial role in several linguistic communication process / NLP tasks, like report grouping, positioning, wistful investigation, etc. Totally various portrayals may catch what's more, unravel various levels of informative fixings covered up inside the content. Along these lines, it has pulled in great and enough amount of consideration from a few specialists, and shifted sorts of models are made arrangements for text outline and preparing. With the outstanding development of online text based data, the best approach to arrange text information viably and with productivity has gotten a pivotal, hard what's more, requesting issue. Text arrangement, a strategy for appointing predefined classes to test archives, is considered as an important tool for handling this issue. To address key challenges associated in this domain, it requires innovative ways of thinking and development of computationally efficient methods to overcome those problems so that the models can be practically deployable in various real-time aspects. 


\section{References}

[1] Charu C. Aggarwal, ChengXiang Zhai (2012), A survey of text classification algorithms., In: Mining text data. Springer, pp. 163-222.

[2] Raymond J. Mooney and Un Yong Nahm, Text Mining with Information Extraction, Proceedings of the 4th International MIDP Colloquium, September 2003, Bloemfontein, South Africa.

[3] D.E. Knuth, On the Translation of Languages from Left to Right, INFORMATION AND CONTROL 8, 607 - 639, (1965).

[4] A.L. Blum and P. Langley. Selection of relevant features and examples in machine learning. Artificial Intelligence, 97:245-271, 1997.

[5] G. Forman. An extensive empirical study of feature selection metrics for text classification. Journal of Machine Learning Research, 3:1289-1305, 2003

[6] I. Guyon and A.Elisseeff. An introduction to variable and feature selection. Journal of Machine Learning Research, 3:1157-1182, 2003.

[7] R. Kohavi and G.H. John. Wrappers for feature subset selection. Artificial Intelligence, 97:273-324, 1997.

[8] D. Fragoudis, D. Meretakis, and S. Likothanassis. Integrating feature and instance selection for text classification. In Proceedings of the $8^{\text {th }}$ Annual ACM SIGKDD Conference, pages 501-506, 2002.

[9] T. Joachims. Text categorization with support vector machines: Learning with many relevant features. In Proceedings of the 10th European Conference on Machine Learning, pages 137-142, 1998.

[10] D. Mladenic and M. Grobelnik. Feature selection for unbalanced class distribution and Naive Bayes. In Proceedings of the 16th International Conference on Machine Learning, pages 258-267, 1999.

[11] Y. Yang. Sampling strategies and learning efficiency in text categorization. In AAAI Spring Symposium on Machine Learning in Information Access, pages 88-95, 1996.

[12] Y.Yang and J.O. Pedersen. A comparative study on feature selection in text categorization. In Proceedings of the 14th International Conference on Machine Learning, pages 412-420, 1997.

[13] H. Kim, P. Howland, and H. Park. Dimension reduction in text classification with support vector machines. Journal of Machine Learning Research, 6:37-53, 2005.

[14] . Leopold and J. Kindermann. Text categorization with support vector machines. How to represent texts in input space? Machine Learning, 46:423-444, 2002.

[15] F. Sebastiani. Machine learning in automated text categorization. ACM Computing Surveys, 34(1):1-47, 2002.

[16] Y. Yang. Noise reduction in a statistical approach to text categorization. In Proceedings of the 18th Annual International ACM SIGIR Conference, pages 256-263, 1995.

[17] Y. Yang and X. Liu. A re-examination of text categorization methods. In Proceedings of the 22nd Annual International ACM SIGIR Conference, pages 42-49, 1999 .

[18] J. Zhang and Y. Yang. Robustness of regularized linear classification methods in text categorization. In Proceedings of the 26th Annual International ACM SIGIR Conference, pages 190-197, 2003.

[19] T. Zhang and F.J. Oles. Text categorization based on regularized linear classification methods. Information Retrieval, 4(1):5-31, 2001.

[20] Yuchen Zhang, Panupong Pasupat, Percy Liang. Macro Grammars and Holistic Triggering for Efficient Semantic Parsing, arXiv.org > arXiv:1707.07806, 2017.

[21] Yiming Li, Baogang Wei, Yonghuai Liu, Liang Yao, Hui Chen, Jifang Yu, Wenhao Zhu. Incorporating knowledge into neural network for text representation, Expert Systems with Applications, Volume 96, pp.103-114, April 2018.

[22] Sebastian Schuster, Matthew Lamm, Christopher D. Manning Gapping Constructions in Universal Dependencies v2, NoDaLiDa 2017, pages 123-132, Gothenburg, Sweden, 22 May 2017.

[23] Lam, W., Ho, C. (1998). Using a generalized instance set for automatic text categorization. SIGIR'98. pp. 81-89.

[24] Tang, Y. H., and Gao, J. H. (2007). Improved classification for problem involving overlapping patterns. In IEICE transaction on information and systems (Vol. E90- D, No.11, pp. 1787-1795).

[25] Sarkar, M. (2007). Fuzzy-rough nearest neighbor algorithms in classification. Fuzzy Sets and Systems, 158(19), 2

[26] Liu, B., Lee, W. S., Yu, P., and Li, X. (2002). Partially supervised classification of text documents. In Proceedings of the 19th international conference on machine learning (pp. 8-12).

[27] Yu, H., Han, J. W., and Chang, K. C.-C. (2004). PEBL: Web page classification without negative examples. IEEE Transactions on Knowledge and Data Engineering, 6(1), 70-81.

[28] Y. Li, S.C.K. Shiu, S.K. Pal, J.N.K. Liu (2006). A rough set-based case-based reasoner for text categorization, International Journal of Approximate Reasoning, 41 (2006) 229-255.

[29] Duoqian Miao, Qiguo Duan, Hongyun Zhang, Na Jiao (2009). Rough set based hybrid algorithm for text classification, Expert Systems with Applications 36 (2009), 9168-9174.

[30] Weibin Deng, A Hybrid Algorithm for Text Classification based on Rough Set, 978-1-61284-840-2, (2011) IEEE.

[31] Ahmed T. Sadiq and Sura Mahmood Abdullah. Hybrid Intelligent Techniques for Text Categorization, 2012 International Conference on Advanced Computer Science Applications and Technologies,2013 IEEE.

[32] Guansong Pang and Shengyi Jiang. A generalized cluster centroid based classifier for text categorization, Information Processing and Management 49 (2013) 576-586.

[33] Basant Agarwal and Namita Mittal. Sentiment Classification using Rough Set based Hybrid Feature Selection, 4th Workshop on Computational Approaches to Subjectivity, Sentiment and Social Media Analysis, pages 115-119, Association for Computational Linguistics, Atlanta, Georgia, 14 June 2013.

[34] Ricardo, Ilias Flaounas, Nello Cristianini. Efficient classification of multi-labeled text streams by clashing, Expert Systems with Nibaran Das, Swarnendu Ghosh, Teresa Goncalves, and Paulo Quaresma. Comparison of Different Graph Distance Metrics for Semantic Text Based Classification, ResearchGate Publications, DOI:10.17562/ PB-49-6, June 2014.

[35] Guo-Niu Zhu, Jie Hu, Jin Qi, Jin Ma, Ying-Hong Peng. An integrated feature selection and cluster analysis techniques for case-based reasoning, Engineering Applications of Artificial Intelligence 39 (2015) 14-22.

[36] Jun Wang, Jiaxu Peng, Ou Liu. A classification approach for less popular webpages based on latent semantic analysis and rough set model, Expert Systems with Applications 42 (2015) 642-648.

[37] Vinod Kumar Bhalla and Neeraj Kumar. An efficient scheme for automatic web pages categorization using the support vector machine, New Review of Hypermedia and Multimedia, (2016), Taylor-Francis online, VOL. 22, NO. 3, 223-242.

[38] Abdullah Saeed Ghareb, Azuraliza Abu Bakar, Abdul Razak Hamdan Hybrid feature selection based on enhanced genetic algorithm for text categorization, Expert Systems with Applications 49 (2016) 31-47 